\title{
Generation of the first BAC-based physical map of the common carp genome
}

\author{
Peng $\mathrm{Xu}^{1{ }^{*+}}$, Jian Wang ${ }^{1+}$, Jintu Wang ${ }^{1,2}$, Runzi Cui ${ }^{1,4}$, Yan $\mathrm{Li}^{1,2}$, Zixia Zhao ${ }^{1}$, Peifeng $\mathrm{Ji}^{1}$, Yan Zhang ${ }^{1}$, Jiongtang $\mathrm{Li}^{1}$ \\ and Xiaowen Sun ${ }^{1,3^{*}}$
}

\begin{abstract}
Background: Common carp (Cyprinus carpio), a member of Cyprinidae, is the third most important aquaculture species in the world with an annual global production of 3.4 million metric tons, accounting for nearly $14 \%$ of the all freshwater aquaculture production in the world. Apparently genomic resources are needed for this species in order to study its performance and production traits. In spite of much progress, no physical maps have been available for common carp. The objective of this project was to generate a BAC-based physical map using fluorescent restriction fingerprinting.

Result: The first generation of common carp physical map was constructed using four- color High Information Content Fingerprinting (HICF). A total of 72,158 BAC clones were analyzed that generated 67,493 valid fingerprints (5.5 $\times$ genome coverage). These BAC clones were assembled into 3,696 contigs with the average length of $476 \mathrm{~kb}$ and a N50 length of $688 \mathrm{~kb}$, representing approximately $1.76 \mathrm{~Gb}$ of the common carp genome. The largest contig contained 171 BAC clones with the physical length of $3.12 \mathrm{Mb}$. There are 761 contigs longer than the N50, and these contigs should be the most useful resource for future integrations with linkage map and whole genome sequence assembly. The common carp physical map is available at http://genomics.cafs.ac.cn/fpc/WebAGCoL/Carp/ WebFPC/.
\end{abstract}

Conclusion: The reported common carp physical map is the first physical map of the common carp genome. It should be a valuable genome resource facilitating whole genome sequence assembly and characterization of position-based genes important for aquaculture traits.

\section{Background}

Common carp (Cyprinus carpio), a member of Cyprinidae, is the third most important aquaculture species in the world with an annual global production of 3.4 million metric tons, accounting for nearly $14 \%$ of the all freshwater aquaculture production in the world [1]. Common carp is mainly cultured in Eurasia continent with a culture history of several thousand years, and it was introduced into Africa and America some two centuries ago. In addition to its aquaculture importance, common carp is also considered as a model species for studies on ecology [2], environmental toxicology [3,4], development [5], immunology [6], evolutionary

\footnotetext{
* Correspondence: xupeng@cafs.ac.cn; sunxw2002@163.com

+ Contributed equally

'The Centre for Applied Aquatic Genomics, Chinese Academy of Fishery Sciences, Beijing, 100141, China

Full list of author information is available at the end of the article
}

genomics [7], nutrition [8], and physiology [3]. As such, great interests exist to generate its genetic and genomic resources. Significant progress has been made recently including a large number of polymorphic genetic markers [6,9-11], linkage maps [12,13], a large number of ESTs (unpublished), a bacterial artificial chromosome (BAC) library [14], a large dataset of BAC-end sequences (BES) [15], and cDNA microarrays [16]. Some of these genomic resources have been used to analyze important genes [17] and quantitative trait loci (QTL) related to various economic traits such as growth rate, cold-tolerance, muscle quality, and amino acid content $[18,19]$. However, no physical maps have been constructed, hindering the progress of whole genome sequencing project as well as genetic improvement programs.

Common carp has a genome size of 1.6-2.0 Gb, as estimated from flow cytometry [20-22]. This is

\section{Biomed Central}


significantly larger than its closely related grass carp (1 Gb). Along with its large genome size, common carp has twice as many chromosomes as most other cyprinid fishes, making many to believe that an additional round of whole genome duplication (4R) may have occurred 50 Myr ago [23-25]. Such potential tetraploidization could add significant challenges to the whole genome sequencing project for common carp, a project currently in progress. Clearly, a physical map is demanded for scaffolding the small sequence contigs into scaffolds, and eventually into chromosome-scale sequence assemblies.

Physical maps have been proven as an important genome resource. A high quality physical map is very useful to understanding of genome structure and organization, and to positional cloning of genes associating to economically important traits. For genome sequencing projects, especially those using the high throughput next generation sequencing platforms, a high quality physical map and enough BAC end sequences are required to make the genome assembly accurately[26-29]. In addition, physical map could be integrated with linkage map by either mapping BACanchored genetic markers into linkage map or locating markers of linkage map on physical map contigs. The integrated map could be used in comparative mapping and genomic analysis of closely related species and enhance the understanding of unsequenced genomes [28,30].

In the past decade, several physical maps have been constructed in aquaculture ray-finned fishes including Nile tilapia (Oreochromis niloticus) [31], Atlantic salmon (Salmo salar) [32], channel catfish (Ictalurus punctatus) [33,34], rainbow trout (Oncorhynchus mykiss) [35] and Asian sea bass (Lates calcarifer) [36]. Here we report the first BAC-based physical map of the common carp genome.

\section{Results and Discussion}

\section{BAC fingerprinting and contig assembly}

Four-color high information content fingerprinting (HICF) [37] was used to generate the restriction fingerprints of all BAC clones from the common carp BAC library. A total of 89,088 BAC clones, representing around 7.3-fold coverage of the common carp genome, were processed. Of these processed BAC clones, a total of 72,158 ( $81 \%$ success) fingerprints were used for the construction of the physical map after removal of low quality fingerprints using FPminer 2.1 [38]. These fingerprints represented approximately 5.9-fold coverage of the common carp genome (Table 1). On average, each BAC clone contained 98.7 restriction bands, with a range of 60 to 120 bands from most of the BACs (Figure 1). Each band represented approximate $1.4 \mathrm{~kb}$ on average, as assessed from the average insert size (141 $\mathrm{kb})$ of the BAC library [14].
The 72,158 valid fingerprints were used for BAC contig assembly. A total of 67,493 BAC clones, representing 5.5 -fold coverage of common carp genome, were assembled into 3,696 contigs. There were 4,665 unassembled BAC clones remaining as singletons. Each contig contains 18.3 BAC clones, with an average length of $476 \mathrm{~kb}$ (Table 1). The contig size distribution is shown in Figure 2. The largest contig contains $171 \mathrm{BAC}$ clones with the physical length of $3.12 \mathrm{Mb}$. The N50 length of this assembly is $688 \mathrm{~kb}$. There are 761 contigs longer than the N50, serving as the most useful resource for future integration with linkage map and whole genome sequence assembly.

There are a total of 1,234,511 consensus bands (CB) in this assembly, representing approximate $1.76 \mathrm{~Gb}$ of the common carp genome $(1,234,511 \mathrm{CB} \times 1.428 \mathrm{~kb}$ per $\mathrm{CB}$ ). Each BAC in the contigs contributes 18.2 distinct bands or $26 \mathrm{~kb}$ linear length to the assembly on average. The physical length of all the assembled BAC contigs is slightly longer than our commonly used estimation $(1.7 \mathrm{~Gb})$ of the genome size of common carp, but shorter than the estimation of Ojima and Yamamoto [20]. We believe that the summed length of all BAC contigs would be shorter than the real genome size as single BAC library cannot possibly cover $100 \%$ of the genome, because there would be some missing genomic regions caused by restriction enzyme bias, leaving gaps in the assembled physical map. However, a real BAC contig could be split into two contigs or more when we use assembly parameters of high stringency, especially for those genome regions with higher levels of heterozygosity.

Questionable clones (Q-clones) generally result from one or several false positive overlaps during physical map assembly. Sometimes, FPC may not be able to assign an appropriate linear order to a specific BAC clone on the consensus map, and marked it as a Qclone. In this study, the function DQer were used to break up all contigs containing over $15 \%$ Q-clones after several rounds of end-to-end merging and single-to-end merging with lowered stringency of cutoff values progressively.

There were a total of 2,812 Q-clone-free contigs (76.1\%) and $884 \mathrm{Q}$-contigs in the final version of the assembly. In the $884 \mathrm{Q}$-contigs, a total of $1,448 \mathrm{Q}-$ clones were presented. However, vast majority of these Q-contigs only contained five Q-clones or less. There were only $12 \mathrm{Q}$-contigs $(0.3 \%)$ containing more than 5 Q-clones (Table 2). The questionable clones could be generated by inconsistency in enzyme digestion, contamination, doubled peaks and possible chimeric contigs during the assembly. In spite of these technical effects, Q-clones could be also caused by the duplication status of common carp genome. Teleost fish genomes are well 
Table 1 Statistics of the physical map assembly of the common carp

\begin{tabular}{lll}
\hline Total number of BAC clones fingerprinted & 89,088 & $\sim 7.3 \times$ genome equivalent \\
Valid fingerprints for FPC assembly & 72,158 & $\sim 5.9 \times$ genome coverage \\
Total number of contigs assembled & 3696 & $\sim 5.5 \times$ genome coverage \\
Clones contained in the 3696 contigs & 67,493 & \\
Average BAC clones per contig & 18.26 & \\
Average contig size in consensus bands (CB) & 334 & \\
Estimated average contig size (kb) & 476 & \\
Estimated N50 contig size (kb) & 688 & \\
Number of Q-contigs & 884 & \\
Number of Q-clones & 1448 & \\
Number of singletons & 4665 & 141 \\
Average insert size of the BAC library (kb) & 98.7 & 18.2 bands per BAC clone in the consensus map \\
Average number of bands per fingerprinted BAC clone & 1.428 & $\sim 1 \times$ genome size \\
Average size each band represents (kb) & $1,234,511$ & $1.76 \mathrm{~Gb}$ \\
Total number of bands included in the contigs & & \\
Total physical length of assembled contigs &
\end{tabular}

known for their whole genome duplication events, and high level of tandem and segmental gene duplications had been observed in teleost species. As common carp may have had an additional round of whole genome duplication (4R). High level of genome duplications would certainly add complexities for physical map assembly and possibly produce Q-clones.

The physical map of common carp is accessed through the web-based FPC viewer at http://genomics. cafs.ac.cn/fpc/WebAGCoL/Carp/WebFPC/.

\section{Assessment of the physical map}

Three approaches were used to assess the reliability of the physical map assembly for common carp. First, eight contigs were randomly selected which contained end-toend merging junction. The primers were designed from $\mathrm{BAC}$ end sequence on one side of the junction site, and used in the PCR on both BAC clones of the junction. The results showed that PCR can successfully amplify positive products on the junctions of eight selected

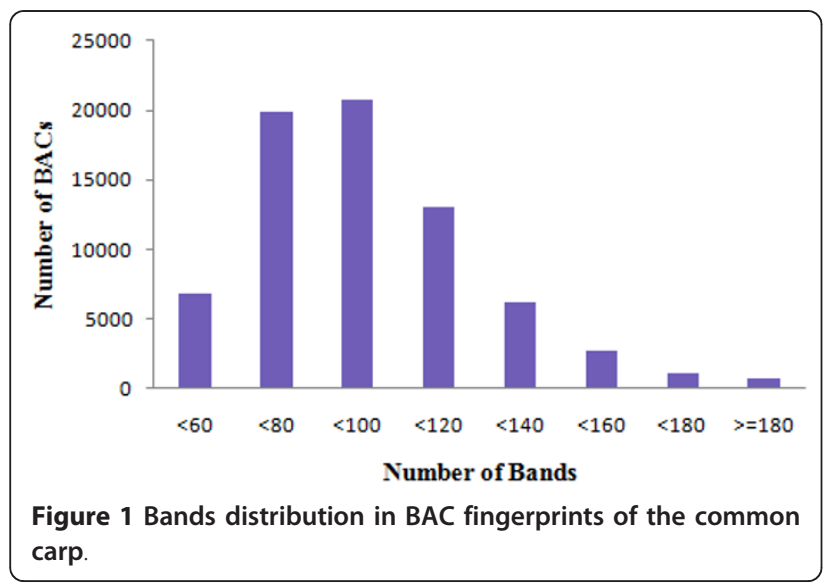

contigs, indicating that both $\mathrm{BAC}$ clones on the junction site were truly overlapped in the common carp genome (Table 3). Since the junction sites were generated by end to end merging with looser stringency, the reliability of the whole contig was then proved. A second approach was also used to assess the physical map assembly from randomly selected contigs. Briefly, eighteen contigs with various lengths were selected randomly. PCR primers were designed from BAC end sequences, and PCR reactions were performed on all BAC clones. For those long contigs, multiple pairs of primers were designed and used, if necessary, to cover all $\mathrm{BAC}$ clones in the contig. If all the BAC clones truly belong to the contig, they should be identified by PCR reactions in the contig, thereby confirming the contig. As shown in Table 4 all of the BAC clones in surveyed eighteen contigs could be positively identified by PCR assays, providing strong evidence for the high reliability of the physical map of common carp. The third approach was used to evaluate the physical map assembly by mapping physical map contigs to common carp

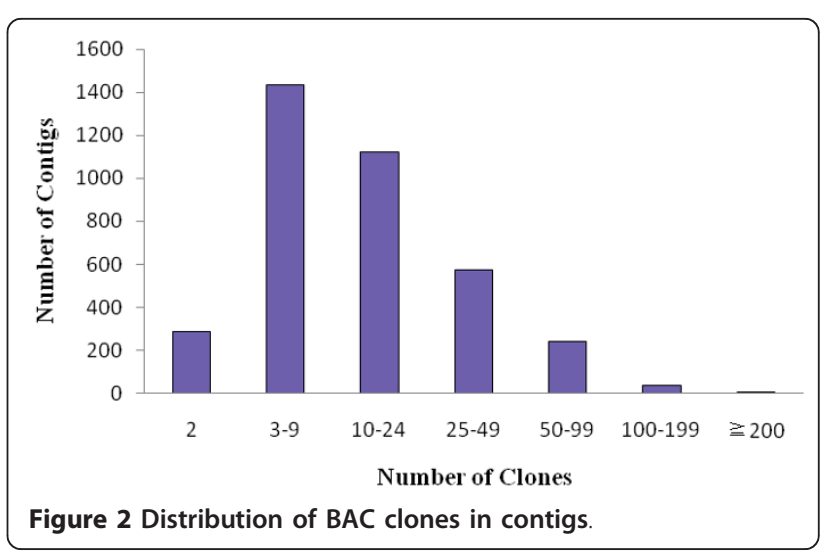


Table 2 Distribution of Q-clones in assembled contigs

\begin{tabular}{ccc}
\hline Number of contigs & Q-clones/contig & Percentage of all contigs \\
\hline 2812 & 0 & 76.1 \\
552 & 1 & 14.9 \\
188 & 2 & 5.1 \\
80 & 3 & 2.2 \\
34 & 4 & 0.9 \\
18 & 5 & 0.5 \\
12 & $>5$ & 0.3
\end{tabular}

Note that $76.1 \%$ of the contigs are free of Q-clones, and most Q-clones are involved in a small number of contigs.

linkage map (Zhang, et al, unpublished). The unpublished linkage map contained 858 microsatellite markers, including 271 BAC-derived microsatellite markers, which make it good resource for the physical map assessment. A total of 179 BAC-derived microsatellite markers had been located on both physical map contigs and linkage map which could serve as anchors for map integration. A total of 7 contigs had been identified that mapped on 7 distinct linkage groups. The genetic distances of two anchor points on the linkage map were ranged from $0.80 \mathrm{cM}$ to $6.39 \mathrm{cM}$ (Table 5). Thus, all of the 7 physical map contigs had been validated using linkage analysis, further validating the physical map assembly of common carp. Figure 3 was an example that common carp physical map contig 251 was anchored to a genetic linkage group 11 by using BACanchored microsatellite markers. More contigs could be mapped on linkage map by developing and anchoring more contig-derived markers on common carp linkage map, which will lead to comprehensive integration of physical and linkage maps gradually.

\section{Conclusion}

Here we reported the construction of the first physical map of the common carp genome. The physical map was constructed with valid fingerprints of 67,493 clones $(5.5 \times$ genome coverage). The physical map can be accessed at http://genomics.cafs.ac.cn/fpc/WebAGCoL/ Carp/WebFPC/. This physical map contained 3,696 contigs with a N50 length of $688 \mathrm{~kb}$. The consensus length of assembled contigs was $1.76 \mathrm{~Gb}$, consistent with the estimated genome size of common carp (1.7 Gb-2.0 Gb). The assembly was validated by using PCR assays on randomly selected contigs and mapping physical map contigs on linkage map. This physical map should be useful for various genome projects of common carp, especially for the currently ongoing whole genome sequencing project of carp.

\section{Methods \\ BAC library}

The Hind III BAC library of common carp used for the construction of the physical map was previously reported [14]. Briefly, the library was made from a female common carp with a total of 92,160 recombinant clones and an average insert size of $141 \mathrm{~kb}$. This library represented approximately 7.6-fold genome coverage of the common carp genome.

\section{BAC DNA isolation and fingerprinting}

BAC clones were inoculated into four 96 deep-well culturing plates using a 96-pin replicator (V\&P Scientific, San Diego, CA, USA). Each well of the 96 deep-well culturing plates contained $1.2 \mathrm{ml} 2 \times \mathrm{YT}$ medium and 12.5 $\mu \mathrm{g} / \mathrm{ml}$ chloramphenicol. The deep-well culturing plates were then covered with air permeable seals (Excel Scientific, Victorville, CA, USA) and incubated at $37^{\circ} \mathrm{C}$ with $300 \mathrm{rpm}$ shaking for 20 hours. BAC DNA was then isolated using a modified alkaline method with lysate clarification using Fritted Filter Plate (NUNC, Roskilde, Denmark). BAC DNA was resuspended in $50 \mu \mathrm{l}$ of milliQ water in 96 -well plates and stored at $-20^{\circ} \mathrm{C}$ before use.

Twenty $\mu$ BAC DNA of each BAC clone was digested by BamHI, EcoRI, XbaI, XhoI, and HaeIII restriction endonucleases (New England Biolabs, Ipswich, MA, USA) at $37^{\circ} \mathrm{C}$ for three hours simultaneously, and then end-labeled using SNaPshot Multiplex kit (Life technologies, Foster City, CA, USA), according to manufacturer's instructions. The 6 -bp cutter restriction

Table 3 Assessment of overlapping reliability at end to end merging points by using PCR

\begin{tabular}{ccc}
\hline \multicolumn{2}{c}{ Clones overlapping each other } & cutoff value of end merging \\
\hline CYC054L01 & CYC121J09 & $1 \mathrm{e}-40$ \\
CYC204K23 & CYC054L01 & $1 \mathrm{e}-40$ \\
CYC027124 & CYC203H04 & $8 \mathrm{e}-37$ \\
CYC096 P15 & CYC148K22 & $4 \mathrm{e}-36$ \\
CYC016K05 & CYC207J08 & $5 \mathrm{e}-25$ \\
CYC013P01 & CYC240P24 & $1 \mathrm{e}-21$ \\
CYC086L08 & CYC118D14 & $4 \mathrm{e}-36$ \\
CYC055K17 & CYC098L15 & $2 \mathrm{e}-28$ \\
+
\end{tabular}$+$


Table 4 Assessment of assembly reliability on randomly selected contigs by using PCR with primers designed from $B A C$ end sequences

\begin{tabular}{ccccc}
\hline Contig ID & Number of primer pairs & Number of Clones & Number of Positive Clones & Contig assembly completely validated \\
\hline 2042 & 15 & 65 & 65 & Yes \\
113 & 12 & 85 & 85 & Yes \\
2092 & 8 & 34 & 34 & Yes \\
348 & 2 & 11 & 11 & Yes \\
4368 & 4 & 11 & 11 & Yes \\
1494 & 4 & 25 & 25 & Yes \\
2220 & 4 & 14 & 14 & Yes \\
4074 & 2 & 10 & 10 & Yes \\
1258 & 2 & 13 & 13 & Yes \\
6064 & 2 & 13 & 13 & Yes \\
3806 & 4 & 17 & 17 & Yes \\
4498 & 4 & 13 & 13 & Yes \\
677 & 6 & 37 & 37 & Yes \\
2995 & 4 & 24 & 24 & Yes \\
3682 & 4 & 22 & 22 & Yes \\
5929 & 2 & 7 & 7 & Yes \\
3097 & 2 & 12 & 12 & Yes \\
984 & 2 & 12 & 12 & Yes \\
\hline
\end{tabular}

endonucleases EcoRI (G'AATTC), XbaI (T'CTAGA), BamHI (G'GATCC), XhoI (C'TCGAG) generate 5'-protruding ends allowing differentially fluorescence labeled A, C, G, and T to be incorporated at the 3' ends of fingerprints while the 4-bp cutter HaeIII cleave the fragments to small segments making them suitable for analysis using an automated sequencer [37]. The labeled BAC fragments were precipitated by using pre-chilled $100 \%$ ethanol following by washing with $70 \%$ ethanol, then suspended in $10 \mu \mathrm{l} \mathrm{Hi-Di} \mathrm{Formamide} \mathrm{and} \mathrm{analyzed}$ with GeneScan 500 LIZ Size Standard on 3730XL DNA Analyzer (Life technologies).

\section{Fingerprint collection and processing}

The fragment sizes in each BAC fingerprint were collected by the Data Collection program on the ABI 3730XL Genetic Analyzer, then processed by software FPminer 2.1 [38]. Briefly, the fragment size calling was conducted using automatic algorithm in FPminer. Several quality control steps were applied to the fingerprints: the empty wells were removed; the off-scale fragments with peak height greater than 6,000 relative fluorescent units (RFU) were removed; the fingerprints with fewer than 50 or more than 250 fragments were removed. Cross-contamination check was also conducted on FPminer to remove potential contaminated clones. In addition, the fingerprints having greater than 60 fragments of any single fluorescent color were also considered as contaminated clones and removed. Vector fragments and high frequency fragments were identified by fragment frequency analysis and then removed in FPminer. The sizes files were then output from FPminer for contig assembly in FPC program (http://www.agcol.arizona.edu/software/fpc).

\section{Contig assembly}

The program FPC version 9.3 was used to assemble the BAC fingerprinting data into BAC contigs. FPC parameters were adjusted for the HICF method as described

Table 5 Validation of physical map assembly by linkage mapping of microsatellites isolated from clones in the common carp physical map.

\begin{tabular}{|c|c|c|c|c|c|}
\hline Contig ID & Number of BAC Clones & Contig Length (kb) & Genetic Distance (cM) & Number of Markers & Linkage Group ID \\
\hline 251 & 55 & 822 & 0.80 & 2 & 11 \\
\hline 285 & 30 & 765 & 1.55 & 2 & 2 \\
\hline 633 & 11 & 237 & 3.35 & 2 & 16 \\
\hline 1769 & 25 & 510 & 4.07 & 2 & 4 \\
\hline 3108 & 19 & 590 & 1.73 & 2 & 1 \\
\hline 3749 & 28 & 520 & 6.39 & 2 & 36 \\
\hline 4017 & 20 & 550 & 1.33 & 2 & 3 \\
\hline
\end{tabular}




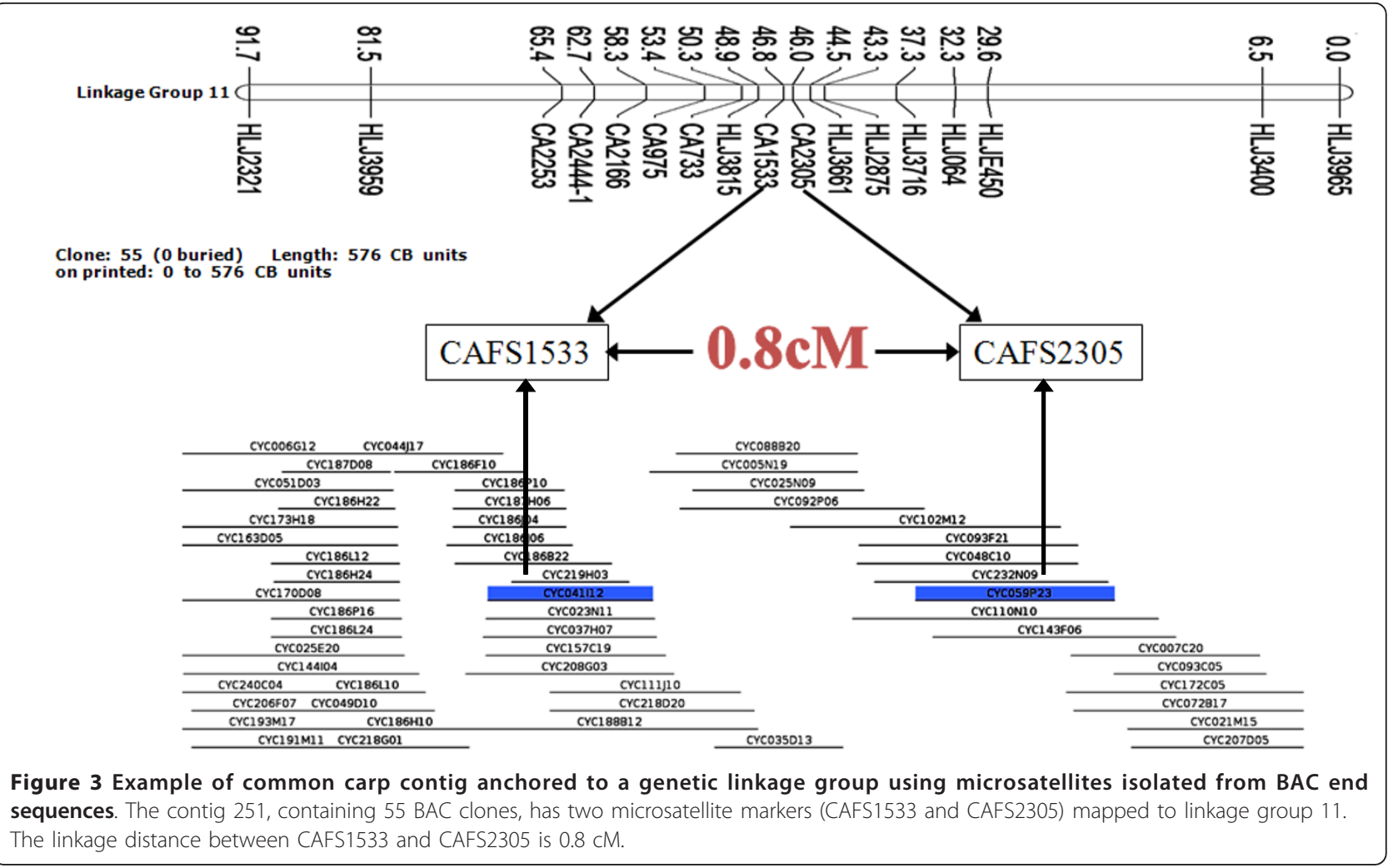

in the tutorials. The size tolerance was set at $0.4 \mathrm{bp}$, and Sulston score cutoff was initially set as 1e-40. After the first round of assembly, the DQer function was performed to break down all contigs more than 15\% of Q clones to eliminate false assembly. Several rounds of end-to-end merging with consecutive reductions of the Sulston score cutoff stringency at 1e-15 were then performed, and followed by single-to-end merging until the final cutoff of 1e-15 was reached.

\section{Contig quality assessment using PCR method}

$\mathrm{BAC}$ contigs were randomly selected, and BAC-end sequences on those selected contigs were used to develop primers for contig validation and reliability examination. Briefly, all BAC clones on the selected contigs were picked from stocking plates and inoculated into culturing plates. BAC DNA was then extracted using alkaline method as we described above. PCR reactions with primers from specific contig were conducted on all BAC clones of the contig in $25 \mu \mathrm{l}$ solution containing $10 \mathrm{ng}$ BAC DNA, $1 \times$ PCR buffer, $100 \mu \mathrm{mol}$ of each dNTPs, $0.2 \mu \mathrm{mol}$ forward primer, $0.2 \mu \mathrm{mol}$ reverse primer and $1 \mathrm{U}$ of Taq DNA polymerase (Fermentas, Glen Burnie, Maryland, USA) on ABI 9700 thermal cycler (Life Technologies) under the following cycling conditions: initial denaturation at $95^{\circ} \mathrm{C}$ for $3 \mathrm{~min}$; then 35 cycles of $94^{\circ} \mathrm{C}$ for $30 \mathrm{sec}, 55^{\circ} \mathrm{C}$ for $30 \mathrm{sec}$ and $72^{\circ} \mathrm{C}$ for $45 \mathrm{sec}$; final extension at $72^{\circ} \mathrm{C}$ for $5 \mathrm{~min}$. All primers were listed in Additional file 1 Table S1. PCR products were then analyzed to detect positive BAC clones using electrophoresis on $1.2 \%$ agarose gel. The BAC clones that supported positive PCR amplification with a single pair of PCR primers were considered to be overlapped.

\section{Contig validation using $\mathrm{BAC}$-anchored microsatellite markers on linkage map}

Microsatellite markers were previously developed from BAC end sequences[15] and genotyped in a F1 common carp family for linkage mapping (Zhang et al, unpublished). The linkage map contained 271 BAC-derived microsatellite markers, which could serve anchor points for physical and linkage map integration. Physical map contigs containing at least one anchor microsatellite markers were then mapped to linkage map. The contigs harboring two or more BAC-anchored microsatellite markers were collected for assembly assessment. Microsatellite markers on one physical map contig should be also mapped to one linkage group with reasonable genetic distance if physical map was assembled correctly.

\section{Additional material}

Additional file 1: Table S1. All primers used for the assessment of common carp physical map. 


\section{Acknowledgements}

This study was supported by the grants from National Department Public Benefit Research Foundation (No. 200903045), National High-tech R\&D Program of China (No. 2009AA10Z105 and 2011AA100401), China Ministry of Agriculture "948" Program (No. 2010-Z11) and Research Foundation of Chinese Academy of Fishery Sciences (No. 2009B002).

\section{Author details}

'The Centre for Applied Aquatic Genomics, Chinese Academy of Fishery Sciences, Beijing, 100141, China. ${ }^{2}$ College of Fisheries and Life Science, Shanghai Ocean University, Shanghai, 201306, China. ${ }^{3}$ Heilongjiang Fisheries Research Institute, Chinese Academy of Fishery Sciences, Harbin, 150070, China. ${ }^{4}$ College of Life Science, Tianjin Normal University, Tianjin, 300387, China.

\section{Authors' contributions}

PX designed and supervised the physical mapping project, and drafted the manuscript. WJ worked on data collection and physical map assembly. JTW, $Y L$ and RC participated in BAC culture and DNA extraction. ZZ worked on BAC library manipulation. YZ participated in microsatellite identification and linkage analysis. PJ and JL worked on bioinformatics analysis and WebFPC. XS supervised the common carp genome project. All authors read and approved the final manuscript.)

Received: 8 August 2011 Accepted: 2 November 2011

Published: 2 November 2011

\section{References}

1. Cultured Aquatic Species Fact Sheets. [http://www.fao.org/fishery/ culturedspecies/search/en].

2. Kulhanek SA, Leung B, Ricciardi A: Using ecological niche models to predict the abundance and impact of invasive species: application to the common carp. Ecological Applications 2011, 21(1):203-213.

3. Van Campenhout K, Bervoets L, Redeker ES, Blust R: A kinetic model for the relative contribution of waterborne and dietary cadmium and zinc in the common carp (Cyprinus carpio). Environmental Toxicology and Chemistry 2009, 28(1):209-219.

4. Kroupova H, Prokes M, Macova S, Penaz M, Barus V, Novotny L, Machova J: Effect of nitrite on early-life stages of common carp (Cyprinus carpio L.). Environmental Toxicology and Chemistry 2010, 29(3):535-540.

5. Liu D, Liu S, You C, Chen L, Liu Z, Liu L, Wang J, Liu Y: Identification and Expression Analysis of Genes Involved in Early Ovary Development in Diploid Gynogenetic Hybrids of Red Crucian Carp $\times$ Common Carp. Marine Biotechnology 2010, 12(2):186-194.

6. Kongchum P, Palti Y, Hallerman EM, Hulata G, David L: SNP discovery and development of genetic markers for mapping innate immune response genes in common carp (Cyprinus carpio). Fish \& Shellfish Immunology 2010, 29(2):356-361.

7. Zhang Y, Liang L, Jiang P, Li D, Lu C, Sun X: Genome evolution trend of common carp (Cyprinus carpio L.) as revealed by the analysis of microsatellite loci in a gynogentic family. Journal of Genetics and Genomics 2008, 35(2):97-103.

8. Gregory M, King H, Bain P, Gibson R, Tocher D, Schuller K: Development of a Fish Cell Culture Model to Investigate the Impact of Fish Oil Replacement on Lipid Peroxidation. Lipids 2011, 1-12.

9. Zhang Y, Liang L, Jiang P, Li D, Lu C, Sun X: Genome evolution trend of common carp (Cyprinus carpio L.) as revealed by the analysis of microsatellite loci in a gynogentic family. J Genet Genomics 2008, 35(2):97-103.

10. Wang D, Liao X, Cheng L, Yu X, Tong J: Development of novel EST-SSR markers in common carp by data mining from public EST sequences. Aquaculture 2007, 271(1-4):558-574.

11. Zhou J, Wu Q, Wang Z, Ye Y: Genetic variation analysis within and among six varieties of common carp (Cyprinus carpio L.) in China using microsatellite markers. Genetika 2004, 40(10):1389-1393.

12. Sun X, Liang L: A genetic linkage map of common carp (Cyprinus carpio L.) And mapping of a locus associated with cold tolerance. Aquaculture 2004, 238(1-4):8

13. Cheng L, Liu L, Yu X, Wang D, Tong J: A linkage map of common carp (Cyprinus carpio) based on AFLP and microsatellite markers. Anim Genet 2010, 41(2):191-198.
14. Li Y, Xu P, Z Zhao, Wang J, Zhang Y, Sun X: Construction and Characterization of the BAC Library for Common Carp Cyprinus Carpio L. and Establishment of Microsynteny with Zebrafish Danio Rerio. Marine Biotechnology 2010.

15. Xu P, Li J, Li Y, Cui R, Wang J, Zhang Y, Zhao Z, Sun X: Genomic insight into the common carp (Cyprinus carpio) genome by sequencing analysis of BAC-end sequences. BMC Genomics 2011, 12:188.

16. Moens LN, van der Ven K, Van Remortel P, Del-Favero J, De Coen WM: Gene expression analysis of estrogenic compounds in the liver of common carp (Cyprinus carpio) using a custom CDNA microarray. $J$ Biochem Mol Toxicol 2007, 21(5):299-311.

17. Wan Y, Zhang Y, Ji P, Li Y, Xu P, Sun X: Molecular characterization of CART, $\mathrm{AgRP}$, and MC4R genes and their expression with fasting and re-feeding in common carp (Cyprinus carpio). Molecular Biology Reports 2011, 1-9.

18. Zhang Y, Xu P, Lu C, Kuang Y, Zhang X, Cao D, Li C, Chang Y, Hou N, Li H, et al: Genetic Linkage Mapping and Analysis of Muscle Fiber-Related QTLs in Common Carp (Cyprinus carpio L.). Marine Biotechnology 2010, 1-17.

19. Mao RX, Liu FJ, Zhang XF, Zhang Y, Cao DC, Lu CY, Liang LQ, Sun XW: [Studies on quantitative trait loci related to activity of lactate dehydrogenase in common carp (Cyprinus carpio)]. Yi Chuan 2009, 31(4):407-411.

20. Ojima Y, Yamamoto K: Cellular DNA contents of fishes determined by flow cytometry. Kromosomo 1990, , II-57: 1871-1888.

21. Tiersch TR, Chandler RW, Wachtel SS, Elias S: Reference standards for flow cytometry and application in comparative studies of nuclear DNA content. Cytometry 1989, 10(6):706-710.

22. Animal Genome Size Database. [http://www.genomesize.com].

23. Ohno S, Muramoto J, Christian L, Atkin NB: Diploid-tetraploid relationship among old-world members of the fish family Cyprinidae. Chromosoma 1967, 23(1):1-9.

24. Larhammar D, Risinger C: Molecular Genetic Aspects of Tetraploidy in the Common Carp Cyprinus carpio. Molecular Phylogenetics and Evolution 1994, 3(1):59-68

25. David L, Blum S, Feldman MW, Lavi U, Hillel J: Recent duplication of the common carp (Cyprinus carpio L.) genome as revealed by analyses of microsatellite loci. Mol Biol Evol 2003, 20(9):1425-1434.

26. Xu P, Wang S, Liu L, Peatman E, Somridhivej B, Thimmapuram J, Gong G, Liu Z: Channel catfish BAC-end sequences for marker development and assessment of syntenic conservation with other fish species. Animal Genetics 2006, 37(4):321-326.

27. Lewin HA, Larkin DM, Pontius J, O'Brien SJ: Every genome sequence needs a good map. Genome Res 2009, 19(11):1925-1928.

28. Liu H, Jiang $Y$, Wang $S$, Ninwichian $P$, Somridhivej B, Xu P, Abernathy J, Kucuktas $\mathrm{H}$, Liu Z: Comparative analysis of catfish BAC end sequences with the zebrafish genome. BMC Genomics 2009, 10:592.

29. Soler L, Conte MA, Katagiri T, Howe AE, Lee BY, Amemiya C, Stuart A, Dossat C, Poulain J, Johnson J, et al: Comparative physical maps derived from BAC end sequences of tilapia (Oreochromis niloticus). BMC Genomics 2010, 11:636.

30. Kucuktas H, Wang S, Li P, He C, Xu P, Sha Z, Liu H, Jiang Yanliang, Baoprasertkul Puttharat, Somridhivej Benjaporn, Wang Yaping, Abernathy Jason, Guo Ximing, Liu Lei, Muir William, Liu Zhanjiang: Construction of genetic linkage maps and comparative genome analysis of catfish using gene-associated markers. Genetics 2009.

31. Katagiri T, Kidd C, Tomasino E, Davis JT, Wishon C, Stern JE, Carleton KL, Howe AE, Kocher TD: A BAC-based physical map of the Nile tilapia genome. BMC Genomics 2005, 6(1):89.

32. Ng SHS, Artieri CG, Bosdet IE, Chiu R, Danzmann RG, Davidson WS, Ferguson MM, Fjell CD, Hoyheim B, Jones SJM, et al: A physical map of the genome of Atlantic salmon, Salmo salar. Genomics 2005, 86(4):396-404.

33. Quiniou SM, Waldbieser GC, Duke MV: A first generation BAC-based physical map of the channel catfish genome. BMC Genomics 2007, 8:40.

34. Xu P, Wang S, Liu L, Thorsen J, Kucuktas H, Liu Z: A BAC-based physical map of the channel catfish genome. Genomics 2007.

35. Palti Y, Luo M-C, Hu Y, Genet C, You F, Vallejo R, Thorgaard G, Wheeler P, Rexroad C: A first generation BAC-based physical map of the rainbow trout genome. BMC Genomics 2009, 10(1):462.

36. Xia JH, Feng F, Lin G, Wang CM, Yue GH: A First Generation BAC-Based Physical Map of the Asian Seabass (Lates calcarifer). PLOS ONE 2010, 5(8): e11974. 
37. Luo MC, Thomas C, You FM, Hsiao J, Ouyang S, Buell CR, Malandro M, McGuire PE, Anderson OD, Dvorak J: High-throughput fingerprinting of bacterial artificial chromosomes using the snapshot labeling kit and sizing of restriction fragments by capillary electrophoresis. Genomics 2003, 82(3):378-389.

38. FPminer. [http://www.bioinforsoft.com].

doi:10.1186/1471-2164-12-537

Cite this article as: Xu et al.: Generation of the first BAC-based physical map of the common carp genome. BMC Genomics 2011 12:537.

Submit your next manuscript to BioMed Central and take full advantage of:

- Convenient online submission

- Thorough peer review

- No space constraints or color figure charges

- Immediate publication on acceptance

- Inclusion in PubMed, CAS, Scopus and Google Scholar

- Research which is freely available for redistribution

Submit your manuscript at www.biomedcentral.com/submit
C Biomed Central 